\title{
Nonadiabatic quantum search algorithms
}

\author{
A. Pérez ${ }^{1}$ and A. Romanelli, ${ }^{1,2}$ \\ ${ }^{1}$ Departament de Física Teórica and IFIC, Universitat de València-CSIC Dr. Moliner 50, 46100-Burjassot, Spain \\ ${ }^{2}$ Instituto de Física, Facultad de Ingeniería, Universidad de la República, C.C. 30, C.P. 11000, Montevideo, Uruguay
}

(Received 7 June 2007; published 20 November 2007)

\begin{abstract}
We present two continuous-time quantum search algorithms similar to the adiabatic search algorithm, but now without the requirement of adiabatic evolution. Both algorithms can find the marked state in a time proportional to $\sqrt{N}$. The behavior of the first algorithm is, essentially, similar to Grover's algorithm, but the second model possesses the important property that one does not need to single out a given time in order to find the searched state. After a well-defined transition time, this second algorithm will converge towards the marked state with a high probability, provided the parameters of the Hamiltonian are chosen appropriately. This convergence shows a resemblance to quantum search algorithms with a fixed point [L. K. Grover, Phys. Rev. Lett. 95, $150501(2005)]$.
\end{abstract}

DOI: 10.1103/PhysRevA.76.052318

PACS number(s): 03.67.Lx, 05.45.Mt, 72.15.Rn

\section{INTRODUCTION}

Quantum computation has attracted the attention of researchers from several different areas [1]. This field of knowledge presents new scientific challenges to learn how to work with quantum properties to obtain more efficient algorithms. However, relatively few quantum algorithms were created; among them, Shor's and Grover's [2,3] algorithms are the best known. Grover's search algorithm locates a marked item out of an unsorted list of $N$ elements in a number of steps proportional to $\sqrt{N}$, instead of $O(N)$ as in the classical case. It performs a unitary transformation of the initial quantum state so as to increase the likelihood that the marked state of interest will be measured at the output (amplification technique). It has been proved that there are neither quantum nor classical algorithms that can perform such an unstructured search faster [4]. For several marked items on the database, a new version has been recently suggested that introduces selective phase shifts of angle $\pi / 3$ [5], which possesses the characteristic feature of convergence towards a fixed point. This algorithm can also be improved by adding two ancilla qubits and performing measurements on them [6]. These algorithms, however, are well suited when the fraction of marked entries in the database is large.

The search algorithm has also a continuous-time version [7] that has been described as the analog of the original Grover algorithm. From this continuous-time version and using the quantum adiabatic theorem, adiabatic search algorithms have been developed [8-10] that consist in introducing a time-dependent Hamiltonian which is flexible with respect to the path from the initial to final Hamiltonians and whose dynamics evolves slowly enough so that it remains always near its instantaneous ground state. They solve the search problem in a time proportional to $\sqrt{N} / \delta$, where $\delta$ is a precision parameter that depends on the energy difference between the two lowest states.

Another way to generate a continuous-time quantum search algorithm [11] has been recently developed that finds a discrete eigenstate of a given Hamiltonian $H_{0}$. This algorithm behaves like Grover's and explicitly shows that the search algorithm is essentially a resonance phenomenon between the initial and searched states [12].
In this work we present two continuous-time search algorithms that are controlled by a time-dependent Hamiltonian, similarly to the case of the quantum adiabatic search algorithm, but now the evolution is nonadiabatic; then, it is not necessary to impose slowness on the dynamics in order to preserve the system in the fundamental state. These algorithms provide new insights to search algorithms: in particular, a connection between the resonant and adiabatic search algorithms or the possibility to generate a new type of search algorithm, which is more robust in the sense that one does not need to pick up a particular instant of time when the measurement has to be performed. In this sense, it can be regarded as a continuous-time counterpart for quantum search algorithms with a fixed point [5].

The paper is organized as follows. In the next two sections we develop the two models of nonadiabatic algorithms. In the last section we draw the conclusions of this work.

\section{NONADIABATIC ALGORITHM I}

Consider $N$ items in the database, each associated with a vector in the complete orthonormal set $\{|n\rangle, n=1,2, \ldots, N\}$ in a Hilbert space. Let us call $|s\rangle$ the unknown searched state that is associated with the marked item belonging to the previous group. We assume that the initial state is the symmetric normalized state

$$
\left|\psi_{0}\right\rangle=\frac{1}{\sqrt{N}} \sum_{n=1}^{N}|n\rangle
$$

and define the two Hamiltonians

$$
\begin{gathered}
H_{0}=I-\left|\psi_{0}\right\rangle\left\langle\psi_{0}\right|, \\
H_{s}=I-|s\rangle\langle s|,
\end{gathered}
$$

where $I$ is the identity matrix, their ground states being $\left|\psi_{0}\right\rangle$ and $|s\rangle$, respectively. The algorithm is built from the timedependent Hamiltonian

$$
H(t)=f(t) H_{0}+g(t) H_{s},
$$

where $f(t)$ and $g(t)$ are time-dependent functions that will be defined later. Notice that $H_{s}$ plays the equivalent role to 
"marking" the searched state in Grover's algorithm. The goal of the search algorithm is to change $\left|\psi_{0}\right\rangle$ into $|s\rangle$ or some approximation thereof, following the dynamics generated by the Schrödinger equation. In this problem, we can restrict the analysis to the two-dimensional space spanned by $|s\rangle$ and $|p\rangle=\frac{1}{\sqrt{N-1}} \sum_{n=1, n \neq s}^{N}|n\rangle$. The wave function is then expressed as

$$
|\psi(t)\rangle=a_{s}(t)|s\rangle+a_{p}(t)|p\rangle
$$

for some $a_{s}(t), a_{p}(t)$ such that $\left|a_{s}(t)\right|^{2}+\left|a_{p}(t)\right|^{2}=1$ with $a_{s}(0)$ $=\sqrt{1 / N}$ and $a_{p}(0)=\sqrt{1-1 / N}$. In the $\{|s\rangle,|p\rangle\}$ basis, we have the following matrix for the Hamiltonian:

$$
H(t)=\frac{1}{N}\left[\begin{array}{cc}
(N-1) f(t) & -\sqrt{N-1} f(t) \\
-\sqrt{N-1} f(t) & f(t)+N g(t)
\end{array}\right] .
$$

The above matrix can be rewritten in the form

$$
H(t)=\frac{1}{2}(f+g) I+\frac{1}{2} \omega(t) \vec{n} \cdot \vec{\sigma},
$$

where ${ }^{1} \omega(t)=\sqrt{(f-g)^{2}+4 f g / N}, \quad \vec{n}=\frac{1}{\omega(t)}(-2 \sqrt{N-1} f / N, 0, f$ $-g-2 f / N)$ is a unitary vector, and $\vec{\sigma}$ stands for the Pauli matrices. Defining $\vec{n}=(\sin \theta, 0, \cos \theta)$, one can easily obtain the functions $f$ and $g$ as a function of $\theta$, with the following result:

$$
\begin{gathered}
f(t)=-\frac{N}{2 \sqrt{N-1}} \omega(t) \sin \theta, \\
g(t)=-\frac{N}{2 \sqrt{N-1}} \omega(t) \cos (\theta+\beta),
\end{gathered}
$$

with $\sin \beta \equiv(2-N) / N$ and $\cos \beta \equiv 2 \sqrt{N-1} / N$.

The first term in Eq. (7) is proportional to the identity and therefore amounts to a common (time-dependent) phase that can be ignored if one only wants to evaluate probabilities. We will concentrate on the second term

$$
H^{\prime}(t)=\frac{1}{2} \omega(t) \vec{n} \cdot \vec{\sigma}
$$

which describes the dynamics of the search algorithm. Note that, as $N$ increases, the norm of this Hamiltonian is bounded by a $O(1)$ constant: indeed, $\vec{n}$ has norm 1 and $\omega(t)$ does not depend on $N$, as shown below, Eq. (21). Due to the trade-off of energy and time in the evolution operator, this is an important issue when discussing the scaling of the searching time with $N$, since this scaling might be hidden on the energy scale of the Hamiltonian, thus leading to a misinterpretation of the performance of the algorithm: one should evaluate an algorithm independently of how powerful the (quantum) computer is.

The eigenvalues of this Hamiltonian are $E_{ \pm}(t)= \pm \frac{1}{2} \omega(t)$, with corresponding time-dependent eigenvectors $\left|E_{+}, t\right\rangle$ $=\left(\cos \frac{\theta}{2}, \sin \frac{\theta}{2}\right)$ and $\left|E_{-}, t\right\rangle=\left(-\sin \frac{\theta}{2}, \cos \frac{\theta}{2}\right)$, with respect to the basis $\{|s\rangle, p\rangle\}$. In this form, it becomes evident that the evolution originating from the Hamiltonian amounts to a (time-

\footnotetext{
${ }^{1}$ We omit in some equations the time dependence of $f, g$, and $\theta$.
}

dependent) rotation in the space spanned by the states $\{|s\rangle, p\rangle\}$ with the goal of maximizing the probability of the $|s\rangle$ state.

The Schrödinger equation in this basis, in units such that $\hbar=1$, becomes

$$
\left[\begin{array}{c}
\frac{d a_{s}(t)}{d t} \\
\frac{d a_{p}(t)}{d t}
\end{array}\right]=-i H^{\prime}(t)\left[\begin{array}{l}
a_{s}(t) \\
a_{p}(t)
\end{array}\right] .
$$

Now we take the following steps: first, we change to a new basis where this Hamiltonian is diagonal; second, we solve the Schrödinger equation in that basis; and third, we return to the original basis, where we are searching the state $|s\rangle$. The state describing the system evolution, $|\psi(t)\rangle$, can be expressed as a combination of the time-dependent eigenstates $\left|E_{+}, t\right\rangle$ and $\left|E_{-}, t\right\rangle$,

$$
\begin{aligned}
|\psi(t)\rangle= & a_{+}(t) \exp \left(-i \int_{0}^{t} E_{+}(t) d t\right)\left|E_{+}, t\right\rangle \\
& +a_{-}(t) \exp \left(-i \int_{0}^{t} E_{-}(t) d t\right)\left|E_{-}, t\right\rangle .
\end{aligned}
$$

We have two expressions for this state: one in the $\{|s\rangle,|p\rangle\}$ basis, Eq. (5), and the other one in the $\left\{\left|E_{+}, t\right\rangle,\left|E_{-}, t\right\rangle\right\}$ basis, Eq. (12). The relation between both basis can be expressed as a relation among its coefficients- that is,

$$
\left[\begin{array}{l}
a_{s}(t) \\
a_{p}(t)
\end{array}\right]=U^{\dagger}(t)\left[\begin{array}{c}
a_{+}(t) \exp \left(-i \int_{0}^{t} E_{+}(t) d t\right) \\
a_{-}(t) \exp \left(-i \int_{0}^{t} E_{-}(t) d t\right)
\end{array}\right],
$$

where

$$
U^{\dagger}(t)=\left(\begin{array}{cc}
\cos \frac{\theta}{2} & -\sin \frac{\theta}{2} \\
\sin \frac{\theta}{2} & \cos \frac{\theta}{2}
\end{array}\right) .
$$

The Schrödinger equation in the new coordinates is

$$
\left[\begin{array}{c}
\frac{d a_{+}(t)}{d t} \\
\frac{d a_{-}(t)}{d t}
\end{array}\right]=-M^{\dagger}(t) U(t) \frac{d U^{\dagger}(t)}{d t} M(t)\left[\begin{array}{l}
a_{+}(t) \\
a_{-}(t)
\end{array}\right],
$$

where $M_{11}(t)=\exp \left[-i \int_{0}^{t} E_{+}(t) d t\right], \quad M_{22}(t)=\exp \left[-i \int_{0}^{t} E_{+}(t) d t\right]$, and $M_{12}(t)=M_{21}(t)=0$. From here, one easily arrives at

$$
\begin{gathered}
\frac{d a_{+}(t)}{d t}=-\Omega(t) a_{-}(t), \\
\frac{d a_{-}(t)}{d t}=\Omega^{*}(t) a_{+}(t),
\end{gathered}
$$

with 


$$
\Omega(t) \equiv-\frac{1}{2} \dot{\theta} \exp \left(i \int_{0}^{t} \omega(t) d t\right)
$$

and $\dot{\theta}=\frac{d \theta}{d t}$. Alternatively, we can rewrite Eq. (18) as

$$
\Omega(t)=\frac{\sqrt{N-1}}{N \omega^{2}}\left(\frac{d g(t)}{d t} f(t)-g(t) \frac{d f(t)}{d t}\right) \times \exp \left(i \int_{0}^{t} \omega(t) d t\right) .
$$

We shall take $f(0)=1$ and $g(0)=0$ in this section; then, the initial conditions in the new coordinates are $a_{+}(0)=0$ and $a_{-}(0)=1$. Up to this point, our treatment of the problem is similar to that of the adiabatic algorithm. Now, to proceed further, we shall choose the function $\theta(t)$ [or, equivalently, the functions $f(t)$ and $g(t)$ ] for the nonadiabatic approach in a way that has similarities with the resonant search algorithm. As seen in Eq. (18), if we choose $\theta(t)$ so as to cancel the time dependence of the modulus of $\Omega$, the system of equations (16) and (17) will have an oscillatory solution, with a period proportional to $\sqrt{N}$, which can be identified with the Grover search time. This situation reminds us of the resonant search algorithm [11], but now these amplitudes are not the amplitudes $a_{s}(t)$ and $a_{p}(t)$.

In order to solve analytically the system of Eqs. (16) and (17) we shall impose the conditions

$$
\begin{gathered}
\frac{d g(t)}{d t} f(t)-g(t) \frac{d f(t)}{d t} \equiv \varepsilon(\alpha t+\gamma)^{2}, \\
\omega(t)=|\alpha t+\gamma| .
\end{gathered}
$$

In these expressions $\varepsilon$ is a coupling parameter between the states $a_{+}(t)$ and $a_{-}(t), \alpha$ is the velocity parameter associated with the energy gap, and $\gamma=1$ is dictated by our choice of $f(0)$ and $g(0)$. One can check that Eqs. (20) and (21) are equivalent to imposing

$$
\dot{\theta}=2 \Omega_{0},
$$

with $\Omega_{0}=\frac{\sqrt{N-1}}{N} \varepsilon$. In this way, conditions (20) and (21) simply imply both a mixing angle $\theta(t)$ and a $g a p \omega(t)$ which evolve linearly with time and thus determine the time evolution of the functions $f(t)$ and $g(t)$; see Eqs. (8) and (9).

We can decouple Eqs. (16) and (17) to obtain a differential equation for $a_{-}(t)$ [and similarly for $\left.a_{+}(t)\right]$ :

$$
\frac{d^{2} a_{-}}{d t^{2}}+i \omega(t) \frac{d a_{-}}{d t}+\Omega_{0}^{2} a_{-}=0 .
$$

This equation can be solved in the same way as was done in [14]. The change of variables,

$$
W=\exp \left[\frac{i}{4}\left(\alpha t^{2}+2 \gamma t\right)\right] a_{-},
$$

leads to

$$
\frac{d^{2} W}{d z^{2}}+\left(\eta+\frac{1}{2}-\frac{1}{4} z^{2}\right) W=0
$$

where

$$
z=\sqrt{\alpha} \exp (-i \pi / 4)(t+\gamma / \alpha)
$$

and

$$
\eta=i \frac{\Omega_{0}^{2}}{\alpha} .
$$

The solutions to Eq. (25) are the parabolic cylinder functions $D_{\eta}(z)$ [15]. In our case we write the general solution as

$$
W(z)=A_{1} D_{\eta}(z)+A_{2} D_{\eta}(-z),
$$

where the coefficients are determined by the initial conditions $a_{-}(0)=1$ and $\frac{d a_{-}(0)}{d t}=0$. These coefficients are

$$
\begin{aligned}
& A_{1}=\frac{D_{\eta-1}\left(-z_{0}\right)}{D_{\eta-1}\left(z_{0}\right) D_{\eta}\left(-z_{0}\right)+D_{\eta}\left(z_{0}\right) D_{\eta-1}\left(-z_{0}\right)}, \\
& A_{2}=\frac{D_{\eta-1}\left(z_{0}\right)}{D_{\eta-1}\left(z_{0}\right) D_{\eta}\left(-z_{0}\right)+D_{\eta}\left(z_{0}\right) D_{\eta-1}\left(-z_{0}\right)},
\end{aligned}
$$

with $z_{0}=\exp (-i \pi / 4) \gamma / \sqrt{\alpha}$. Finally, the amplitude $a_{+}(t)$ can be calculated using the above result for $a_{-}(t)$ and Eq. (17).

Let us discuss in more detail the qualitative behavior of the results we have obtained so far. For large $N$ and finite $\varepsilon$ such that $\Omega_{0} \simeq \frac{\varepsilon}{\sqrt{N}} \ll 1$, it follows from Eqs. (24)-(30) that $\left|a_{-}(t)\right| \simeq 1$ and $\left|a_{+}(t)\right| \simeq 0$. On the other hand, for large $N$ one can approximate $\theta \simeq 2 \Omega_{0} t$. Then, using Eq. (13), the following approximation for the probabilities of the searched and the orthogonal states are obtained:

$$
\begin{aligned}
& P_{s}(t) \simeq \sin ^{2}\left(\Omega_{0} t\right), \\
& P_{p}(t) \simeq \cos ^{2}\left(\Omega_{0} t\right),
\end{aligned}
$$

which are valid whenever $t$ satisfies $(\alpha t+\gamma)>0$. Note that Eqs. (31) and (32) are independent of the value of $\alpha$ if the previous conditions are verified. Then, if we let the system evolve during a time $\tau \equiv \frac{\pi \bar{N}}{2 \varepsilon}$ and we measure immediately after that, the probability to obtain the searched state is equal to 1 . In this case, our method behaves qualitatively like Grover's. The parameter $\varepsilon$ allows for a faster search (relatively to the standard Grover's algorithm): one can even obtain a characteristic time $\tau \sim 1$. This speedup is allowed because the energy scale in the Hamiltonian, defined by the functions $f(t)$ and $g(t)$, is large enough [cf. Eq. (22) in [13]], provided that $\Omega_{0} \ll 1$. For concreteness, we will adopt the value $\varepsilon=1$. Additionally, we have recovered our interpretation of the search algorithm as a quantum resonance between states [11,16-18]; now, the resonance is between the searched and the orthogonal states.

The above result shows that the nonadiabatic algorithm works correctly for $\alpha>0$ (remember that $\gamma=1$ ). We have verified this situation for several values of $N$ and $\alpha>0$, using the exact equation, in Fig. 1. The figure shows a periodic behavior with the Grover characteristic time and the correctness of the approximation in Eqs. (31) and (32) as $N$ increases.

When $\alpha=0$ [see Eq. (23)], $a_{-}$and $a_{+}$can be easily obtained analytically: 


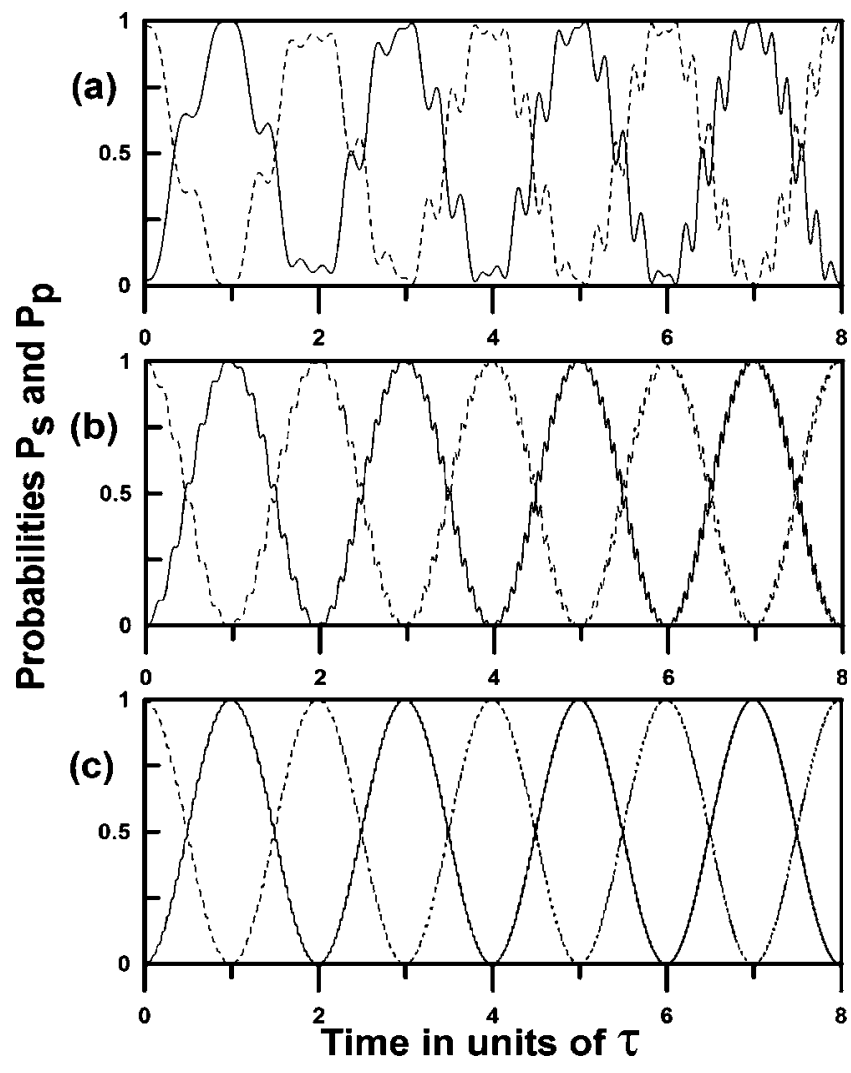

FIG. 1. Probabilities, as a function of time, in units of the characteristic time $\tau \equiv \frac{\pi \sqrt{N}}{2 \varepsilon}$. The solid line corresponds to the searched state $P_{s}$, the dashed line to the orthogonal state $P_{p}$. In all cases, $\alpha$ $>0$, the probabilities being independent of the value of $\alpha$. The sizes of the searched set are (a) $N=50$, (b) $N=500$, and (c) $N=5000$.

$$
\begin{gathered}
a_{-}(t)=\exp \left(-i \frac{\gamma}{2} t\right)\left[\cos \phi(t)+\frac{i \gamma}{\sqrt{\left(\frac{\gamma}{2}\right)^{2}+\Omega_{0}^{2}}} \sin \phi(t)\right] \\
a_{+}(t)=\frac{\Omega_{0} \exp \left(-i \frac{3 \gamma}{2} t\right)}{\sqrt{\left(\frac{\gamma}{2}\right)^{2}+\Omega_{0}^{2}}} \sin \phi(t),
\end{gathered}
$$

with $\phi(t)=\sqrt{\left(\frac{\gamma}{2}\right)^{2}+\Omega_{0}^{2}} t$. From these expressions and for large $N, \Omega_{0} \ll 1,\left|a_{+}(t)\right| \simeq 0$ and $\left|a_{-}(t)\right| \simeq 1$, and using the same arguments as before, it can be shown that the search algorithm also works in this case. For $\alpha<0$ the behavior of the system is quite more complex. If, additionally, $|\alpha| \simeq 0$ (then both $\left|z_{0}\right|$ and $|z|$ go to infinity) using Eqs. (13) and (26)-(30) and the asymptotic properties of the parabolic cylinder functions, it can be shown that $P_{-}(t)=\left|a_{-}(t)\right|^{2} \simeq 1$ as before; then, for large $N$ and $\Omega_{0}<1$, Eqs. (31) and (32) are again obtained, and the search algorithm continues to operate. For the case $\alpha<0$ but finite we shall use another reasoning that could have also been used in the previous cases. Notice that the characteristic frequencies of the probability amplitudes $\left(\sim \Omega_{0}\right)$ are, in general, very small compared to the timedependent characteristic frequency of $\Omega(t)$-i.e., $\frac{1}{t} \int_{0}^{t} \omega(t) d t$; then, the stationary phase method can be used to integrate approximately the differential equations (16) and (17) for $\alpha$ $\neq 0$. We have used this method in the case $\alpha<0$ and $\alpha$ finite to obtain Eqs. (31) and (32) with the condition $t<t_{c} \equiv-\frac{\gamma}{\alpha}$. The time $t_{c}$ is the "close approach time," defined as the time when the derivative of the phase of $\Omega(t)$ vanishes [see Eq. (18)] and at the same time the energy levels cross each other. Then the search algorithm operates up to this time if $\Omega_{0}$ $\ll \frac{1}{t} \int_{0}^{t} \omega(t) d t$. For times $t \geqslant t_{c}$ our previous argument cannot be applied and the periodicity of the behavior is not clear because other frequencies are present. This figure establishes that, as $|\alpha|$ is decreased, the close approach time $t_{c}$ increases; in the limit, the algorithm works for all times.

To close this section, let us remark that the previous results can also be obtained without the use of the mobile base in the following way: Let us substitute the expressions of $f(t)$ and $g(t)$ given by the Eqs. (8) and (9) in Eq. (11). These equations, together with the normalization of the wave function and the conditions of maximization of the amplitude of the searched state, allow us to find the time for which the probability of the searched state is maximum. The maximization conditions are $\left|\frac{d a_{s}(t)}{d t}\right|=0,\left|a_{s}(t)\right|=1$, and as a result, we obtain that they are satisfied for those values of $\tau$ such that

$$
f(\tau)=\frac{N(\alpha \tau+\gamma)}{2 \sqrt{N-1}} \cos \left(2 \Omega_{0} \tau+\beta\right)=0 .
$$

From this equation it is deduced that, if $\alpha \tau+\gamma \neq 0$, then

$$
\tau=\frac{\pi N}{2 \sqrt{N-1} \epsilon} l
$$

with $l=1,2,3, \ldots$. As a result, we see that the algorithm works in an equivalent way to the Grover algorithm for all times if $\alpha$ is positive, and only up to the close approach time if $\alpha$ is negative. Figure 2 was obtained using the exact results of this paper. It shows the probability $P_{s}$ for several values of $\alpha$ and also that the approximation made in Eqs. (31) and (32) remains valid for times $t<t_{c}$.

\section{NONADIABATIC ALGORITHM II}

In this section we introduce a new idea for the searching Hamiltonian, which is based on a different choice of the functions $f(t)$ and $g(t)$ and possesses the important property that one does not need to single out a given time in order to find the searched state with a high probability, provided the parameters of the Hamiltonian are chosen appropriately. This means that the time evolution converges towards a fixed point; i.e., it converges to a state which can be chosen to be close to the marked state.

Let us return to Eq. (6) and choose now

$$
f(t)=1
$$

then, we have 


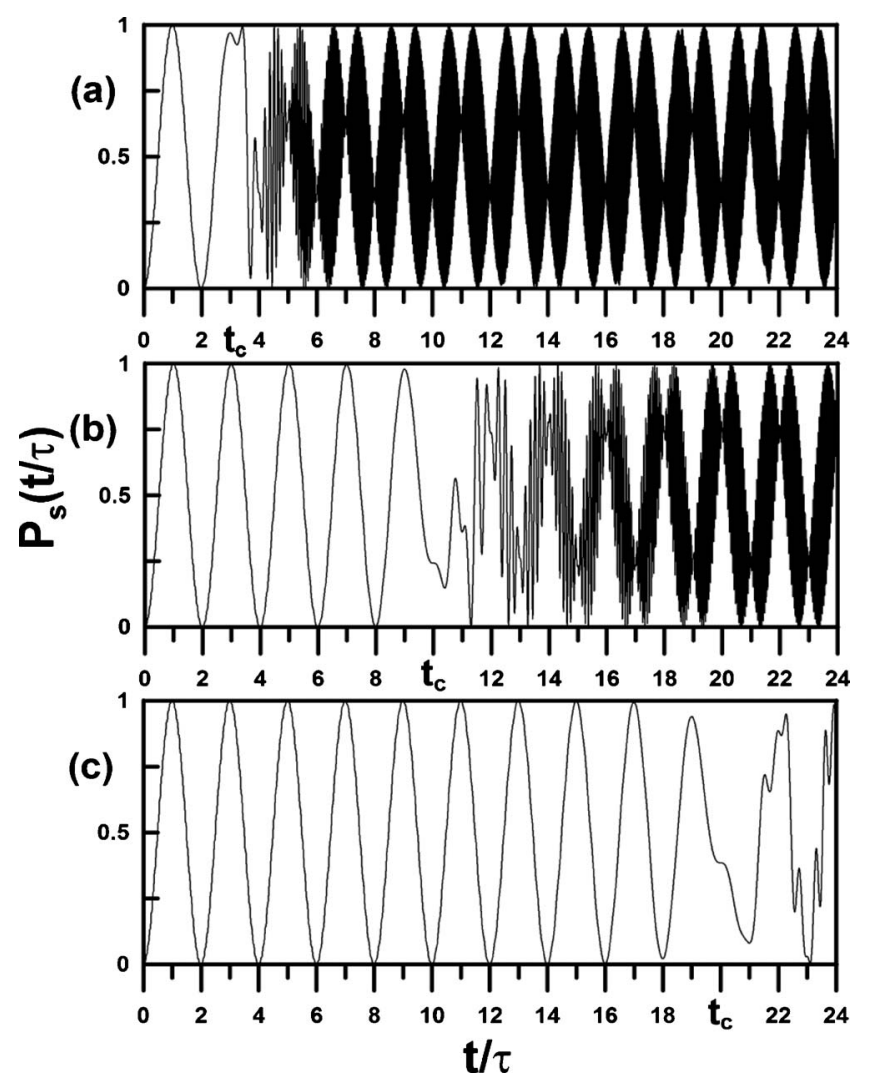

FIG. 2. Probability of the searched state $P_{s}$ as a function of time. $N=5000$. (a) $\alpha=-0.31 \gamma / \tau$, (b) $\alpha=-0.10 \gamma / \tau$, and (c) $\alpha=-0.05 \gamma / \tau$. Note that the values $t_{c}$ change inversely with $\alpha$.

$$
H(t)=\frac{N-1}{N} I+\frac{\sqrt{N-1}}{N}\left[\begin{array}{cc}
0 & -1 \\
-1 & \frac{N g(t)}{\sqrt{N-1}}+\frac{2-N}{\sqrt{N-1}}
\end{array}\right] .
$$

The first term in this expression is constant and proportional to the identity. One can, as done in the previous section, ignore it for the sake of solving the Schrödinger equation. Let us choose the $(2,2)$ matrix element in Eq. (38) so that it changes linearly with time. In this way, the resulting Hamiltonian (in the fixed basis) mimics the evolution of the functions $a_{+}(t)$ and $a_{-}(t)$ obtained in Sec. II. To be more precise, we choose

$$
g(t)=\frac{N-2}{N}+2 \frac{\sqrt{N-1}}{N}(b-c t),
$$

with $b$ and $c$ arbitrary constants. Note that, from the expressions of $f$ and $g$, the norm of the Hamiltonian is again bounded. In this second model, the gap energy function takes the simple form $\omega(t)=\frac{2}{\sqrt{N}} \sqrt{(c t-b)^{2}+1}$ for $N$ large.

With the above definitions, apart from a global phase which we will ignore, the Hamiltonian Eq. (38) gives rise to the same evolution as the matrix

$$
H^{\prime}(t)=\frac{\sqrt{N-1}}{N}\left[\begin{array}{cc}
0 & -1 \\
-1 & 2(b-c t)
\end{array}\right] .
$$

We will allow time to run from $t=0$ to arbitrarily large values $(t \rightarrow \infty)$. As we observe, the above Hamiltonian bears a close resemblance to the usual ones introduced in adiabatic quantum computations, in the sense that it has a time variation which is linear in time. However, in our case we will not start from the ground state of the Hamiltonian; nor will we intend to force the system to be driven to its ground state for some finite time $T$ by making use of the adiabatic theorem.

In order to solve the Schrödinger equation we make the following change of variable from $t$ to $t^{\prime}=\frac{\sqrt{N-1}}{N} t$. The resulting evolution equations for $a_{s}\left(t^{\prime}\right)$ and $a_{p}\left(t^{\prime}\right)$ are

$$
\left[\begin{array}{c}
\frac{d a_{s}\left(t^{\prime}\right)}{d t^{\prime}} \\
\frac{d a_{p}\left(t^{\prime}\right)}{d t^{\prime}}
\end{array}\right]=-i\left[\begin{array}{cc}
0 & -1 \\
-1 & 2\left(b-a t^{\prime}\right)
\end{array}\right]\left[\begin{array}{l}
a_{s}\left(t^{\prime}\right) \\
a_{p}\left(t^{\prime}\right)
\end{array}\right],
$$

where $a$ is a new arbitrary parameter. They can be easily decoupled, leading to

$$
\frac{d^{2} a_{s}}{d t^{\prime 2}}+2 i\left(b-a t^{\prime}\right) \frac{d a_{s}}{d t^{\prime}}+a_{s}=0 .
$$

This equation has to be supplemented with the initial conditions $a_{s}(0)=\sqrt{1 / N}$ and $\frac{d a_{s}}{d t^{\prime}}\left(t^{\prime}=0\right)=i a_{p}(0)=i \sqrt{(N-1) / N}$. With the substitution

$$
W=a_{s} \exp \left[-i\left(\frac{1}{2} a t^{\prime 2}-b t^{\prime}\right)\right],
$$

we arrive at the same equation as in Eq. (25), but now $z$ $=\sqrt{2 a i}\left(t^{\prime}-b / a\right)$ and $\eta=-\frac{i}{2 a}$. The solution to this equation can still be written in the form of Eq. (28), with coefficients $A_{1}$ and $A_{2}$ which have to be determined from the initial conditions. After some algebra, we arrive at

$$
\begin{aligned}
& A_{1}=\frac{1}{\sqrt{N}} \frac{D_{\eta-1}\left(-z_{0}\right)+\sqrt{N} q_{0} D_{\eta}\left(-z_{0}\right)}{D_{\eta-1}\left(z_{0}\right) D_{\eta}\left(-z_{0}\right)+D_{\eta}\left(z_{0}\right) D_{\eta-1}\left(-z_{0}\right)}, \\
& A_{2}=\frac{1}{\sqrt{N}} \frac{D_{\eta-1}\left(z_{0}\right)-\sqrt{N} q_{0} D_{\eta}\left(z_{0}\right)}{D_{\eta-1}\left(z_{0}\right) D_{\eta}\left(-z_{0}\right)+D_{\eta}\left(z_{0}\right) D_{\eta-1}\left(-z_{0}\right)},
\end{aligned}
$$

where $q_{0}=\sqrt{2 a} \sqrt{\frac{N-1}{N}} \exp (i 3 \pi / 4)$ and $z_{0}=-b \sqrt{\frac{2}{a}} \exp (i \pi / 4)$. In order to give a result for the searched probability $P_{s}(t)$ $=\left|a_{s}(t)\right|^{2}$, we need to particularize the values of $N, a$, and $b$. Using the asymptotic form for the parabolic cylinder functions [15], one can obtain the following result for the limit $\lim _{t \rightarrow \infty} P_{s}(t)$ :

$$
p(a, b)=\lim _{t \rightarrow \infty} P_{s}(t)=\left|A_{1} e^{k \pi / 4}+A_{2} e^{-3 \pi k / 4}\right|^{2},
$$

with $k=\frac{1}{2 a}$.

Figure 3 shows a contour plot of the limiting values of the probability $p(a, b)$ with $N=100$. As readily seen, it reveals a complicated pattern with bands of high-probability and lowprobability valleys. These patterns depend quite weakly on 


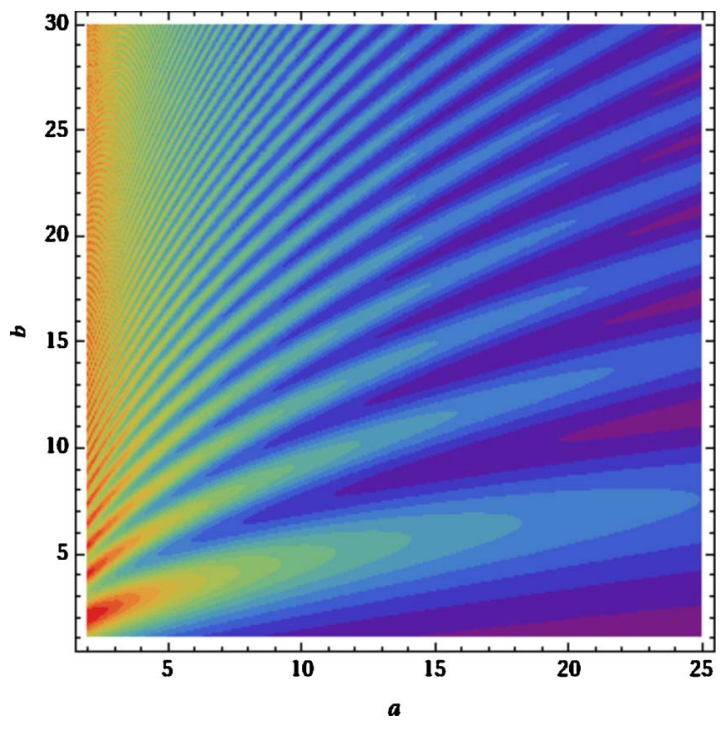

FIG. 3. (Color online) Contour plot for the limiting probability $p(a, b)$ when $N=100$. Following the rainbow colors, red regions represent a probability close to 1 , while purple regions indicate a probability close to 0 .

$N$, and in fact, it is possible to obtain the limit $N \rightarrow \infty$ in Eqs. (44) and (45). Figure 4 corresponds to this limit. As can be seen, the changes are moderate, showing that the asymptotic probability saturates for large values of $N$.

In order to explicitly show the differences between our proposal and the adiabatic treatment, we have calculated the probability $P_{s}(t)$ for different parameters. The results are shown in Fig. 5. Given the structure seen in Fig. 4, we choose a fixed $b=4.5$ and plot the probability for three values of $a: 1,5$, and 20 . The value of $N$ we used is $10^{6}$ which, according to the previous discussion, is equivalent to taking $N \rightarrow \infty$.

In this figure, it is apparent that a transition occurs at the time $t_{c}^{\prime}=b / a$ (corresponding to a value of $t=t_{c} \sim \frac{b}{a} \sqrt{N}$ ), asso-

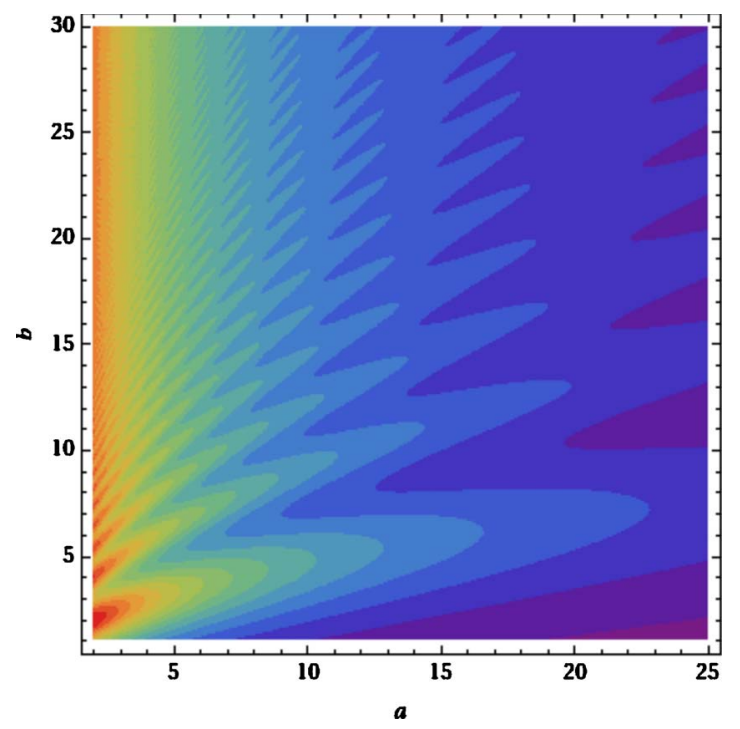

FIG. 4. (Color online) Same as Fig. 3, for the asymptotic $N$ $\rightarrow \infty$ limit.

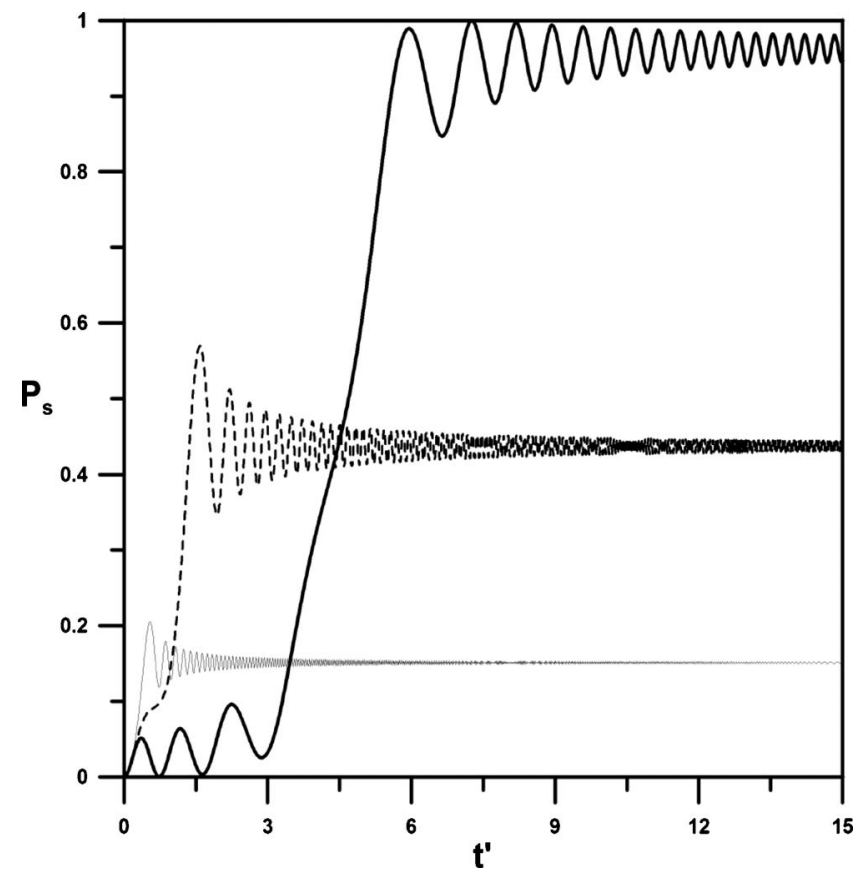

FIG. 5. Evolution of the probability for the searched state $P_{s}$ as a function of the variable $t^{\prime}\left(=\frac{\sqrt{N-1}}{N} t\right)$ for a fixed $b=4.5$ and three values of the parameter $a$ : 1 (solid line, $t_{c} \sim 4.5 \sqrt{N}$ ), 5 (thick dashed line, $t_{c} \sim 0.9 \sqrt{N}$ ), and 20 (thin dashed line, $t_{c} \sim 0.22 \sqrt{N}$ ).

ciated with the point of minimum distance between the two eigenvalues of the Hamiltonian. The asymptotic behavior with time is clear from this figure, although the final probability strongly depends on the particular choice of $a$ and $b$, as seen before.

Therefore, our proposal consists on implementing the Hamiltonian (6), but with the functions $f$ and $g$ as defined above. By an appropriate choice of the parameters $a$ and $b$, one can make the probability of the searched state to reach a value close to unity within a scale of time $O(\sqrt{N})$, as in the usual Grover's algorithm. There exists, of course, the possibility to reduce the standard searching time by increasing the energy scale in the Hamiltonian, as has been discussed by several authors $[13,19]$. However, as already mentioned in the previous section, this is not convenient for the understanding of how powerful the algorithm can be.

Our algorithm, therefore, has the same efficiency as the original one by Grover. Nevertheless, the important result shown in this section is that the evolution drives the system towards a fixed point. This property is also discussed in recent variations on the Grover algorithm [5], using selective phase shifts of $\pi / 3$, which can be improved by adding two ancilla qubits and performing measurements on them [6]. These algorithms are designed for problems where the number of marked states is a significant fraction of the total number of entries in the database. The number of queries to the oracle depends on both the fraction of marked items and the desired precision to find one of this states, and it is shown that they do not represent an improvement over the standard quantum search algorithm when the number of marked states is small, which is the case discussed in this paper. Finally, it 
is important to point out that, when more than one state are marked, this algorithm can be generalized. To this purpose, it is enough to build $|s\rangle$ as an equally weighted linear superposition of the unknown searched states with the initial condition $a_{s}(0)=\sqrt{M / N}$, where $M$ is the number of solutions of the problem. Then, the evolution between the initial state $\left|\psi_{0}\right\rangle$ and the final state $|s\rangle$ is obtained in a time proportional to $\sqrt{N / M}$, which is the known result for the search algorithm for multiple marked states. In this case, if a measurement is made at that precise moment, the system collapses to one of the states that conform $|s\rangle$

\section{CONCLUSIONS}

We have developed two continuous-time quantum search algorithms using a time-dependent Hamiltonian in a nonadiabatic regime that performs the search in a time $O(\sqrt{N})$. Our approach differs from to the usual (adiabatic) algorithm, when one starts from the initial ground state and tries to evolve slowly, making use of the adiabatic theorem to stay close to the instantaneous ground state. For the first case, the key of the algorithm is that the derivatives of the amplitudes $a_{-}(t)$ and $a_{+}(t)$ have a fast time variation with a vanishing mean value over the characteristic time $\tau$-i.e., $\frac{1}{\tau} \int_{0}^{\tau} \frac{d a_{ \pm}(t)}{d t} d t$ $\sim 0$. Then, starting from the ground state, in the mobile basis $\left\{\left|E_{+}, t\right\rangle,\left|E_{-}, t\right\rangle\right\}$, the system remains near this ground state, on average, for all times. This algorithm behaves like the Grover algorithm for non-negative values of the parameter $\alpha$, independently of its particular value, for a large $N$ and $\Omega_{0} \lll 1$. The optimal search time is proportional to $\sqrt{N}$, and the probability to find the searched state oscillates periodically. For $\alpha<0$ the algorithm does not work if $t \geqslant t_{c}$, with $t_{c}$ the close approach time.

The second algorithm makes use of a Hamiltonian that changes linearly with time. In our proposal, the initial and final states do not correspond to the ground states of the Hamiltonian and the system is allowed to evolve up to arbitrarily large times, showing a convergence towards a final state after a finite transition time. When the parameters are chosen appropriately, the asymptotic state can overlap with the searched state with high probability, and one does not need to pick up a special value of time to perform the measurement in order to obtain the desired result. This means that the algorithm will converge towards a fixed point, instead of showing a limit cycle, as in the traditional quantum search algorithm. In this sense, the algorithm is equally efficient as the standard quantum search algorithm and more robust against possible uncertainties in the time when the final measurement has to be performed. However, one has to take into account the fact that, except for a very restricted set of parameters $a$ and $b$ in the Hamiltonian, the asymptotic probability can be close to, but not exactly equal to, unity. It would be interesting to think of some combination of this algorithm, which guarantees a fixed point property, with some amplification technique in order to make the final probability arbitrarily close to unity.

These results open the possibility for the design of new quantum algorithms that perform a search on an unstructured database (and possibly other algorithmic tasks) alternatively to the existing algorithms. It would also be interesting to explore with these algorithm structured search problems within a continuous-time approach-e.g., [20,21].

\section{ACKNOWLEDGMENTS}

We acknowledge comments made by V. Micenmacher and support from PEDECIBA and PDT S/C/OP/28/84. This work has also been supported by the Spanish Ministerio de Educación y Ciencia through Projects Nos. AYA2004-08067C01 and FPA2005-00711.
[1] M. Nielssen and I. Chuang, Quantum Computation and Quantum Information (Cambridge University Press, Cambridge, England, 2000).

[2] P. W. Shor (unpublished); SIAM J. Comput. 26, 1484 (1997).

[3] L. K. Grover (unpublished); Phys. Rev. Lett. 79, 325 (1997).

[4] M. Boyer, G. Brassard, P. Høyer, and A. Tapp, Fortschr. Phys. 46, 493 (1998)

[5] L. K. Grover, Phys. Rev. Lett. 95, 150501 (2005).

[6] T. Tulsi, L. K. Grover, and A. Patel, e-print arXiv:quant-ph/ 0505007.

[7] E. Farhi and S. Gutmann, Phys. Rev. A 57, 2403 (1998).

[8] E. Farhi, J. Goldstone, S. Gutmann, and M. Sipser, e-print arXiv:quant-ph/0001106.

[9] E. Farhi, J. Goldstone, S. Gutmann, and D. Nagaj, e-print arXiv:quant-ph/0512159.

[10] J. Roland and N. J. Cerf, Phys. Rev. A 65, 042308 (2002).

[11] A. Romanelli, A. Auyuanet, and R. Donangelo, Physica A 360, 274 (2006).
[12] L. K. Grover and A. M. Sengupta, Phys. Rev. A 65, 032319 (2002).

[13] S. Das, R. Kobes, and G. Kunstatter, J. Phys. A 36, 2839 (2003).

[14] C. Zener, Proc. R. Soc. London, Ser. A 137, 696 (1932).

[15] I. S. Gradshteyn and I. M. Ryzhik, Table of Integrals, Series and Products (Academic Press, San Diego, CA, 1994).

[16] A. Romanelli, A. Auyuanet, and R. Donangelo, Physica A 375, 133 (2007).

[17] A. Romanelli and R. Donangelo, Physica A 383, 309 (2007).

[18] A. Romanelli, Physica A 379, 545 (2007).

[19] Zhaohui Wei and Mingsheng Ying, e-print arXiv:quant-ph/ 0412117.

[20] E. Farhi, J. Goldstone, and S. Gutmann, e-print arXiv:quant$\mathrm{ph} / 0702144$.

[21] A. M. Childs, B. W. Reichardt, R. Spalek, and S. Zhang, e-print arXiv:quant-ph/0703015. 